This is the author's final, peer-reviewed manuscript as accepted for publication. The publisher-formatted version may be available through the publisher's web site or your institution's library.

\title{
Effects of van der Waals density functional corrections on trends in furfural adsorption and hydrogenation on close- packed transition metal surfaces
}

Bin Liu, Lei Cheng, Larry Curtiss, Jeffrey Greeley

\section{How to cite this manuscript}

If you make reference to this version of the manuscript, use the following information:

Liu, B., Cheng, L., Curtiss, L., \& Greeley, J. (2014). Effects of van der Waals density functional corrections on trends in furfural adsorption and hydrogenation on closepacked transition metal surfaces. Retrieved from http://krex.ksu.edu

\section{Published Version Information}

Citation: Liu, B., Cheng, L., Curtiss, L., \& Greeley, J. (2014). Effects of van der Waals density functional corrections on trends in furfural adsorption and hydrogenation on close-packed transition metal surfaces. Surface Science, 622, 51-59.

Copyright: @ 2013 Elsevier B.V.

Digital Object Identifier (DOI):10.1016/j.susc.2013.12.001

Publisher's Link: http://www.sciencedirect.com/science/article/pii/S0039602813003518

This item was retrieved from the K-State Research Exchange (K-REx), the institutional repository of Kansas State University. K-REx is available at http://krex.ksu.edu 
Manuscript (Surface Science, 2013)

\title{
Effects of van der Waals density functional corrections on trends in furfural adsorption and hydrogenation on close- packed transition metal surfaces
}

\author{
Bin Liu ${ }^{1}$, Lei Cheng ${ }^{2}$, Larry Curtiss ${ }^{2,3}$, and Jeffrey Greeley ${ }^{4 *}$ \\ ${ }^{1}$ Department of Chemical Engineering, Kansas State University, Manhattan, KS 66506 \\ ${ }^{2}$ Materials Science Division, Argonne National Laboratory, Argonne, IL 60439 \\ ${ }^{3}$ Center for Nanoscale Materials, Argonne National Laboratory, Argonne, IL 60439 \\ ${ }^{4}$ School of Chemical Engineering, Purdue University, West Lafayette, IN 47907 \\ ${ }^{*}$ To whom correspondence should be addressed \\ Email: jgreeley@purdue.edu
}

Phone: (765) 494-1282 


\begin{abstract}
The hydrogenation of furfural to furfuryl alcohol on $\operatorname{Pd}(111), \mathrm{Cu}(111)$ and $\operatorname{Pt}(111)$ is studied with both standard Density Functional Theory (DFT)-GGA functionals and with van der Waals-corrected density functionals. VdW-DF functionals, including optPBE, optB88, and optB86b, and Grimme's method, are used to optimize the adsorption configurations of furfural, furfuryl alcohol, and related intermediates resulting from hydrogenation of furfural, and the results are compared to corresponding values determined with GGA functionals, including PW91 and PBE. On Pd(111) and Pt(111), the adsorption geometries of the intermediates are not noticeably different between the two classes of functionals, while on $\mathrm{Cu}(111)$, modest changes are seen in both the perpendicular distance and the orientation of the aromatic ring with respect to the planar surface. In general, the binding energies increase substantially in magnitude as a result of van der Waals contributions on all metals. In contrast, however, dispersion effects on the kinetics of hydrogenation are relatively small. It is found that activation barriers are not significantly affected by the inclusion of dispersion effects, and a Brønsted-EvansPolanyi relationship developed solely from PW91 calculations on $\operatorname{Pd}(111)$ is capable of describing corresponding results on $\mathrm{Cu}(111)$ and $\operatorname{Pt}(111)$, even when the dispersion effects are included. Finally, the reaction energies and barriers derived from the dispersion-corrected and pure GGA calculations are used to plot simple potential energy profiles for furfural hydrogenation to furfuryl alcohol on the three considered metals, and an approximately constant downshift of the energetics due to the dispersion corrections is observed.
\end{abstract}

Keywords: furfural, hydrogenation, periodic density functional theory, van der Waals density functional theory, linear scaling relationship, Brønsted-Evans-Polanyi relationship 


\section{Introduction}

Furfural is central to a variety of catalytic processes associated with biomass chemistry. It can be directly derived from biomass sources and can be further converted to useful chemicals and fuels via hydrogenation and hydrodeoxygenation (HDO). In this context, a model reaction that has received significant attention is the hydrogenation of furfural to furfuryl alcohol [1-8]. In spite of a substantial amount of study, however, furfural hydrogenation catalysts, most of which are based on transition metals or metal alloys, are still incompletely understood, and the hydrogenation process remains the subject of a significant amount research [2, 7, 9-11]. As is the case for numerous other hydrogenation chemistries, such as the selective hydrogenation of acetylene [12], the key catalytic imperative is to maintain a balanced reaction activity and selectivity. This task is particularly challenging in the case of furfural, however, given the presence of multiple conjugated bonds and oxygen-containing functional groups.

Density Functional Theory (DFT) calculations have, in recent years, become standard tools to explore heterogeneous catalytic reaction pathways and related thermodynamic and kinetic properties [13-15]. In particular, when coupled with correlations such as Brønsted-Evans-Polanyi (BEP) relationships and scaling relationships, DFT calculations are extremely powerful for screening, evaluating, and predicting the catalytic properties of a wide range of systems [16-18]. Nevertheless, DFT calculations using the standard Generalized Gradient Approximation (GGA) may be insufficient to accurately describe the weak dispersion effects associated with van der Waals interactions [19], and this problem is especially apparent in the adsorption of highly conjugated organic compounds on precious metals. For example, the adsorption energy of pyridine on $\mathrm{Au}(111)$ is underestimated using PW91 or PBE functionals although these functionals provide reasonable descriptions of adsorbate configurations [20]. An additional example is provided by the work of Bilić et al, who showed, using DFT and CASSCF calculations, that on copper, silver and gold surfaces, the magnitudes of the binding energies of benzene adsorption are significantly underestimated when dispersion effects are neglected [21].

Recent theoretical developments have provided means to partially address the above problems by correcting the total energies from GGA calculations through addition of contributions from non-local density functionals [22-25]. Using DFT calculations and semiempirical corrections based on a QM/MM approach, Tonigold et al were able to determine adsorption energies of benzene and pyridine in good agreement with experiments on $\mathrm{Au}(111)$ [26]. Calculations using the PBE-D3 method of dispersion correction have also been extended recently to mechanistic studies of furfural conversion on Pd [27]. In spite of these advances, however, trends-based analyses that focus on how dispersion forces impact the thermochemistry and kinetics of surface reactions are still relatively rare, and this contribution seeks to provide such trends for the specific case of furfural hydrogenation. 
We report on periodic DFT calculations of adsorption energies, adsorbate geometries, and hydrogenation barriers associated with furfural hydrogenation using several vdW-DF functionals, including optPBE, optB88, optB86b, as well as Grimme's method. Results are given for $\mathrm{Pd}(111), \operatorname{Pt}(111)$, and $\mathrm{Cu}(111)$ surfaces, and the impact of the dispersion corrections, as compared to GGA (PW91 and PBE) results, on the energetics and geometries is described. A simple correlation to permit more rapid estimation of furfural adsorption energetics in these and related systems is presented, and finally, a unified Brønsted-Evans-Polanyi relationship for the PW91 and optB86b functionals is developed.

\section{Computational methods}

Periodic DFT calculations are performed using the Vienna Ab initio Simulation Package (VASP 5.2) [28, 29], where the ionic cores are described by the projector augmented wave (PAW) method [30, 31]. The Kohn-Sham valence states are expanded in the plane wave basis sets up to $340 \mathrm{eV}$. The self-consistent iteration is converged up to $1 \times 10^{-6}$. The ionic step is converged when the force on each atom is less than $0.02 \mathrm{eV} / \AA$. The Methfessel-Paxton smearing scheme is used [32], with a Fermi population of the Kohn-Sham states at $k_{B} T=0.2 \mathrm{eV}$. The total energies are then extrapolated to $0 \mathrm{~K}$.

Several functionals in the vdW-DF family are used in this study, including vdW-DF functionals, such as optPBE, optB88 [23], and optB86b [33], developed by Klimes et al, where the dispersion correlations depend on the density functional in the form of nonlocal correlation terms. We have also performed some tests using Grimme's PBE-D2/D3 method [25], which relies on predetermined $\mathrm{C}_{6}$ coefficients, on $\mathrm{Pd}$ and $\mathrm{Cu}$ surfaces. The PBE-D2 method is used to optimize bulk lattice constants and structures of adsorbed configurations of furfural and furfuryl alcohol, while single point calculations with the PBE-D3 method are used to calculate the binding energies based on both these geometries and geometries optimized with the PBE-GGA [35] functional (bulk cohesive energies are evaluated with PBE-D2 only). In addition to PBE, the standard GGA-PW91 [34] functional is also employed. The reaction barriers for BEP relationship development are calculated using PW91, and optB86b functionals, which we take to be generally representative of the non-dispersion and dispersion correction cases, respectively.

A three-layer, $p(4 \times 4)$ slab, which is sufficiently large to minimize the lateral interactions of adsorbates and their periodic images, is used as the surface model. Adsorption is allowed on only one side of the slab, corresponding to a surface coverage of 1/16 ML. The top layer of the slab is allowed to relax, while the bottom layers are fixed at the appropriate bulk lattice constant. A vacuum spacing equivalent to five metal layers is used between any successive metal slabs. For $\mathrm{Pd}, \mathrm{Cu}$ and $\mathrm{Pt}$ bulk relaxations, a $16 \times 16 \times 16$ Monkhorst-Pack $k$-point mesh is employed [36]. The surface Brillouin zone is sampled with a $2 \times 2 \times 1 k$-point mesh for Pd and Pt, and a $4 \times 4 \times 1 k$-point mesh for $\mathrm{Cu}$. Based on these parameters, we estimate that the binding energies used in this paper have converged to within $50 \mathrm{meV}$. The total energies of relevant gas phase species are

calculated using the same functionals in a box with dimensions of $25 \times 26 \times 27 \AA$; the gamma-point is used for these calculations, with a Gaussian smearing parameter is 0.01 
eV. Spin polarization is used for all gas phase calculations and for surface-adsorbed species with unpaired electrons. Dipole corrections are included in all calculations.

Energy barriers are calculated using the Climbing Image Nudged Elastic Band (CINEB) [37, 38] method on three-layer slabs (top layer relaxed) [39, 40]. The dimer method is then used to further refine the TS obtained from the CINEB calculations. Each transition state has been confirmed to have only one imaginary (negative) vibrational mode.

\section{Results and discussion}

\subsection{Bulk properties of $\mathrm{Pd}, \mathrm{Cu}$, and $\mathrm{Pt}$}

Bulk lattice constants have been optimized for each DFT functional in a four-atom fcc cell; the resulting values are listed in Table 1. The PW91 and PBE functionals overestimate the lattice parameters for both $\mathrm{Pd}$ and $\mathrm{Pt}$. The lattice parameters are generally slightly smaller with vdW-DF functionals, giving better agreement with experimental values [41]. Overall, optB88 and optB86b perform modestly better than the other functionals. We note that the calculated lattice parameters using vdW-DF functionals in this study are in good agreement with the theoretical values reported by Klimes et al. [33] The bulk lattice constants (for $\mathrm{Pd}$ and $\mathrm{Cu}$ ) optimized with the PBE-D2 method are also in good agreement with the experimental value compared with the GGA functionals.

Table 1 Lattice parameters (in $\AA$ ) and cohesive energies (in eV) of bulk Pd, Pt, and $\mathrm{Cu}$ from PW91, PBE, optPBE, optB88, optB86b, and PBE-D2 functionals.

\begin{tabular}{cccc|ccc}
\hline \multirow{2}{*}{$\begin{array}{c}\text { GGA } \\
\text { functionals }\end{array}$} & \multicolumn{3}{c|}{ lattice constant } & \multicolumn{3}{c}{ cohesive energy } \\
\cline { 2 - 7 } & $\mathrm{Pd}$ & $\mathrm{Pt}$ & $\mathrm{Cu}$ & $\mathrm{Pd}$ & $\mathrm{Pt}$ & $\mathrm{Cu}$ \\
\hline PW91 & 3.95 & 3.99 & 3.64 & 3.76 & 6.87 & 3.54 \\
PBE & 3.95 & 3.98 & 3.60 & 3.74 & 6.61 & 3.49 \\
\hline vdW-DF & & & & & & \\
functionals & & & & & & \\
optPBE & 3.97 & 3.97 & 3.65 & 3.78 & 5.58 & 3.44 \\
optB88 & 3.94 & 3.96 & 3.62 & 4.03 & 5.83 & 3.60 \\
optB86b & 3.91 & 3.93 & 3.60 & 4.24 & 6.14 & 3.79 \\
PBE-D2 & 3.91 & - & 3.57 & 4.37 & -- & 3.92 \\
Expt.[41] & $\mathbf{3 . 8 9}$ & $\mathbf{3 . 9 2}$ & $\mathbf{3 . 6 1}$ & $\mathbf{3 . 8 9}$ & $\mathbf{5 . 8 4}$ & $\mathbf{3 . 4 9}$ \\
\hline
\end{tabular}

The cohesive energies are calculated as $E_{\mathrm{coh}}=E_{\text {atom }}-E_{\text {bulk }} / 4$. For Pd, the PW91 and PBE functionals are able to predict the cohesive energies to within $0.2 \mathrm{eV}$ of the experimental values. The agreement with experiment is generally good for optPBE, optB88, and optB86b. The cohesive energies predicted by the PBE-D2 method are larger than the experimental values by approximately $0.5 \mathrm{eV}$ for $\mathrm{Pd}$ and $\mathrm{Cu}$. 


\subsection{Thermochemistry of hydrogenation intermediates}

We begin by describing the calculated geometries of furfural and furfuryl alcohol in the gas phase and on $\operatorname{Pd}(111), \operatorname{Pt}(111)$, and $\mathrm{Cu}(111)$. We then briefly summarize corresponding results for adsorption of other intermediates resulting from furfural hydrogenation. Finally, we present a simple, bond order-based scaling relationship that provides a useful framework for interpreting and extrapolating the calculated adsorption energies of furfural hydrogenation intermediates.

\subsubsection{Geometries of gas phase furfural and furfuryl alcohol}

Figure 1 illustrates the calculated gas phase geometries of furfural and furfuryl alcohol, including both cis and trans configurations. The optimized structures, obtained with all of the DFT functionals, are given in the Supporting Information, where it is seen that the trans configuration is, in all cases, more energetically favored by approximately $0.03 \sim 0.05 \mathrm{eV}$. This result is consistent with the fact that the trans configuration has been observed in IR and Raman spectroscopy studies [42].
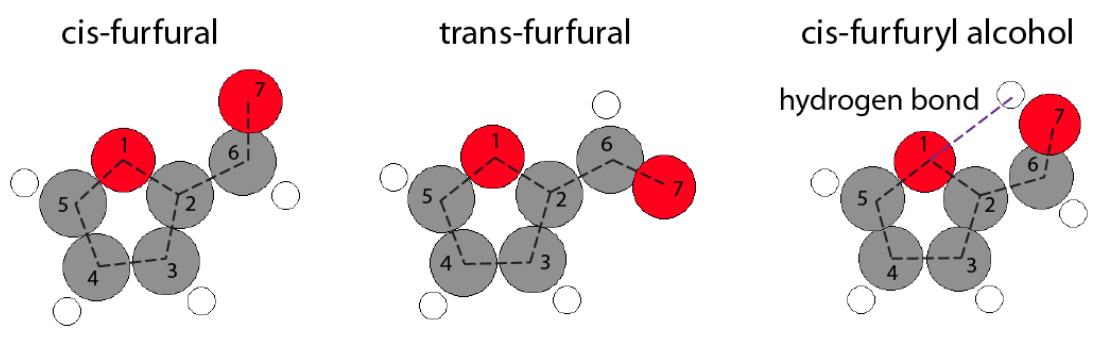

trans-furfuryl alcohol

Fig. 1. Gas phase furfural and furfuryl alcohol in cis and trans configurations. Grey, white, and red spheres represent $\mathrm{C}, \mathrm{H}$, and $\mathrm{O}$ atoms, respectively. The same labels are used throughout the text to identify different atomic sites in furfural, furfuryl alcohol, and their intermediates. Black dashed lines represent covalent $\mathrm{C}-\mathrm{C}$ or $\mathrm{C}-\mathrm{O}$ bonds, and purple dashed lines represent hydrogen bonds between atom $1(\mathrm{O})$ and the hydrogen in the hydroxyl group.

For furfuryl alcohol, the cis configuration is more stable by $0.04 \sim 0.05 \mathrm{eV}$ than the trans configuration; this result may be due to the existence of a weak intramolecular hydrogen bond between the hydroxyl group and the $\mathrm{O}_{1}$ atom in the ring in the cis geometry. The detailed energy differences between the cis and trans configurations for furfural and furfuryl alcohol are given in the Supporting Information.

\subsubsection{Furfural and furfuryl alcohol adsorption}

Adsorption configurations and site preferences can, in general, profoundly affect reaction selectivities $[43,44]$, and these configurations may, in turn, be sensitive to the choice of density functional. To probe these effects, we have optimized the structures of furfural and furfuryl alcohol on $\operatorname{Pd}(111), \mathrm{Cu}(111)$ and $\mathrm{Pt}(111)$ using both dispersion- 
corrected and GGA functionals; the lowest energy configurations for furfural and furfuryl alcohol are shown in Fig. 2 and Fig. 3, respectively. For all metals, the preferred furfural configuration is trans, while for furfuryl alcohol, the cis configuration is preferred on $\mathrm{Cu}(111)$.
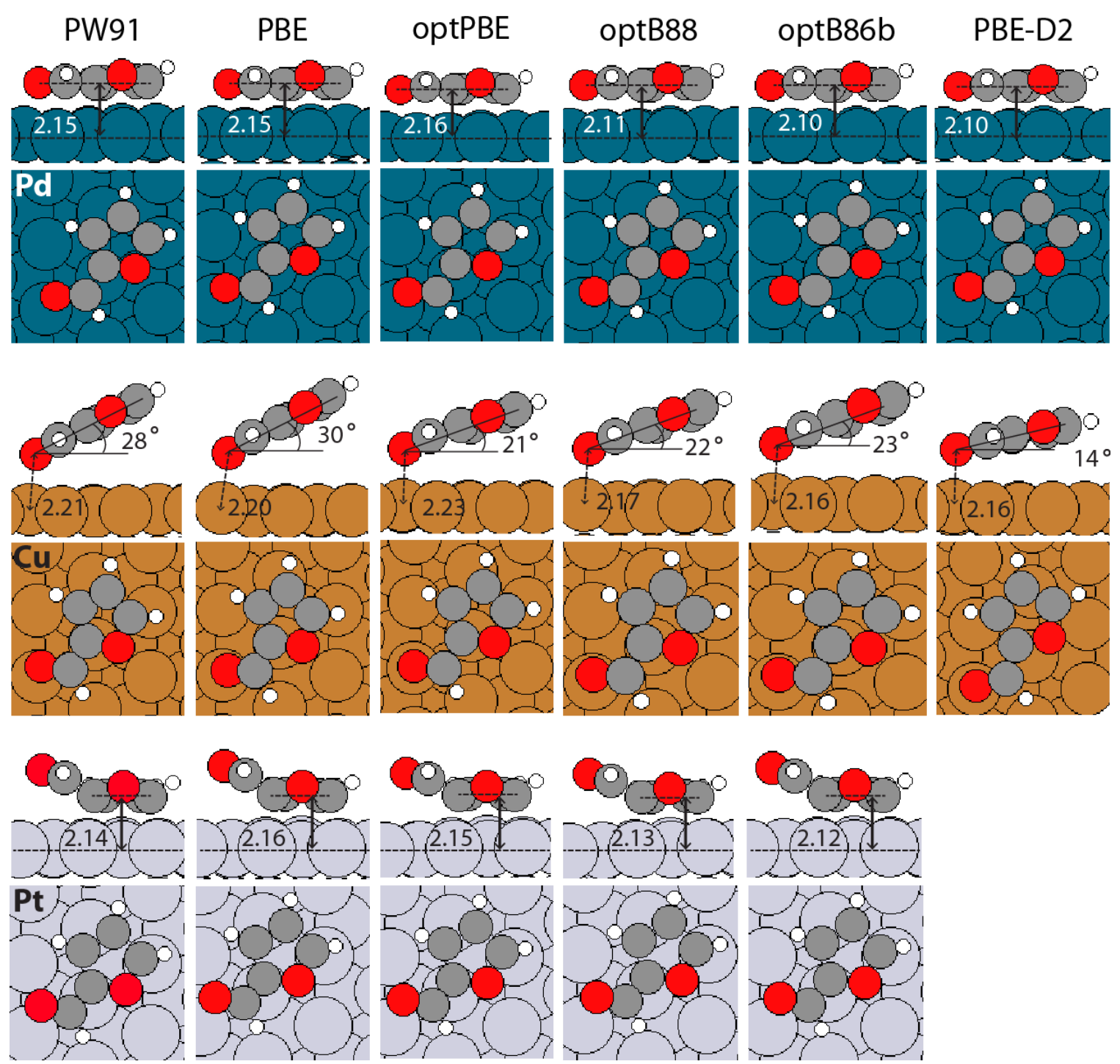

Fig. 2. Adsorption geometries of furfural on $\mathrm{Pd}(111), \mathrm{Cu}(111)$ and $\mathrm{Pt}(111)$, as determined using PW91, PBE, optPBE, optB88, optB86b, and PBE-D2 functionals. Grey, white, and red spheres represent $\mathrm{C}, \mathrm{H}$, and $\mathrm{O}$ atoms, respectively. On $\mathrm{Pd}(111)$, the perpendicular distances of furan ring to the surface are shown. On $\mathrm{Cu}(111)$, the distances between $\mathrm{O} 7$ in the aldehyde group and the nearest $\mathrm{Cu}$ atom are given. On $\operatorname{Pt}(111)$, the perpendicular distances of furan ring to the surface are depicted.

On $\operatorname{Pd}(111)$, little variation is seen among the adsorption geometries of furfural obtained from the different functionals. The adsorbed furfural is parallel to the surface, 
indicating that both the aldehyde group and the five-membered aromatic ring bind to the surface through unsaturated $\mathrm{C}=\mathrm{C}$ and $\mathrm{C}=\mathrm{O}$ bonds. The $\mathrm{C}=\mathrm{O}$ bond in the aldehyde group binds on the off-bridge site. The aromatic ring is centered at the three-fold fcc site [45] so that conjugated $\mathrm{C}=\mathrm{C}$ bonds can bind to either the bridge site or to the Pd top site. On $\operatorname{Pd}(111)$, similar furfural adsorption geometries have been recently reported by Vorontnikov et al using the PBE-D3 functional [27]. We further observe that, although the adsorption geometries do not change significantly due to dispersion effects, the binding energies, defined as $B E=E_{a d s^{*}}-E_{*}-E_{a d s(g)}$ (Table 2), are strongly affected. Indeed, an increase in binding strength of more than $1 \mathrm{eV}$ is seen for optB86b compared to the GGA functionals.

$\mathrm{On} \mathrm{Cu}(111)$, the adsorbed furfural (trans configuration) is in a tilted position $\left(\eta^{1}\right)$. The aldehyde group is in contact with the top site, and the aromatic ring of furfural points away from the surface, as shown in Fig. 2. For different vdW-DF functionals, the angle between the ring and $\mathrm{Cu}(111)$ surface varies, with the configuration optimized with the PBE-D2 functional found to be most closely parallel to the surface. In previous studies, it has been pointed out that the tilted configuration may facilitate preferential hydrogenation of the aldehyde group, thus providing a simple geometrical argument for the known selectivity of furfural hydrogenation to furfuryl alcohol on $\mathrm{Cu}$-based catalysts [43].

The furfural binding energies on $\mathrm{Cu}(111)$ are, in general, significantly weaker than those on $\operatorname{Pd}(111)$ (Table 2). Thus, the relative contribution from dispersion effects is more significant than is the corresponding contribution on $\operatorname{Pd}(111)$. Indeed, the binding energies on $\mathrm{Cu}(111)$ are effectively zero with the GGA functionals, but dispersioncorrected functionals, such as optB86b functional, have binding energies with magnitudes as high as $\sim 0.90 \mathrm{eV}$.

On $\operatorname{Pt}(111)$, furfural (cis configuration) adsorbs primarily via its aromatic ring on three-fold sites. Interestingly, the aldehyde group is tilted away from the surface, suggesting that the largest contribution to the binding energy may come from the interaction of the unsaturated $\mathrm{C}=\mathrm{C}$ bonds in the ring with the $\mathrm{Pt}(111)$ surface. This trend is consistent across all of the functionals. We note that trends in calculated binding energies are similar to those observed for furfural adsorption on $\operatorname{Pd}(111)$, but the binding on $\operatorname{Pt}(111)$ is generally about $\sim 0.2 \mathrm{eV}$ stronger than the corresponding binding on $\operatorname{Pd}(111)$. 
Table 2 Binding energies (in eV) of furfural and furfuryl alcohol on $\operatorname{Pd}(111), \mathrm{Cu}(111)$ and Pt(111) using PW91, PBE, optPBE, optB88, optB86b, and PBE-D2/D3 functionals.

\begin{tabular}{ccccccc}
\hline GGA & \multicolumn{3}{c}{ furfural } & \multicolumn{3}{c}{ furfuryl alcohol } \\
\cline { 2 - 7 } functional & $\mathrm{Pd}$ & $\mathrm{Cu}$ & $\mathrm{Pt}$ & $\mathrm{Pd}$ & $\mathrm{Cu}$ & $\mathrm{Pt}$ \\
\hline PW91 & -0.90 & -0.14 & -1.14 & -1.00 & -0.21 & -1.40 \\
PBE & -0.93 & -0.07 & -1.06 & -0.99 & -0.15 & -1.31 \\
\hline vdW-DF & & & & & & \\
functionals & & & & & & \\
optPBE & -1.49 & -0.66 & -1.69 & -1.62 & -0.78 & -1.96 \\
optB88 & -1.69 & -0.66 & -1.86 & -1.84 & -0.74 & -2.16 \\
optB86b & -2.09 & -0.75 & -2.20 & -2.15 & -0.90 & -2.51 \\
PBE-D2 & -2.38 & -0.90 & ---- & -2.46 & -1.10 & ---- \\
PBE-D3/PBE & -1.97 & -0.74 & --- & -2.06 & -0.85 & --- \\
PBE-D3/PBE-D2 & -1.99 & -1.04 & ---- & -2.11 & -1.24 & --- \\
\hline PBE-D3
\end{tabular}

PBE-D3 energy at PBE geometry

${ }^{\mathrm{b}} \mathrm{PBE}-\mathrm{D} 3$ energy at PBE-D2 geometry

Adsorption configurations of furfuryl alcohol on $\mathrm{Pd}(111), \mathrm{Cu}(111)$, and $\operatorname{Pt}(111)$, optimized with different functionals, are shown in Fig. 3. On $\operatorname{Pd}(111)$, the trans configuration is energetically more stable than similar cis configuration by approximately $0.04 \mathrm{eV}$, and the adsorption occurs primarily through the $\mathrm{C}=\mathrm{C}$ bonds in the aromatic ring at the bridge and top sites. These geometric trends are essentially independent of the particular choice of functional. However, as with furfural, the effect of dispersion corrections on binding energies is generally substantial, with optPBE, optB88 and optB86b giving changes in binding energies of $\sim 0.6 \mathrm{eV}$ compared to the GGA functionals. 


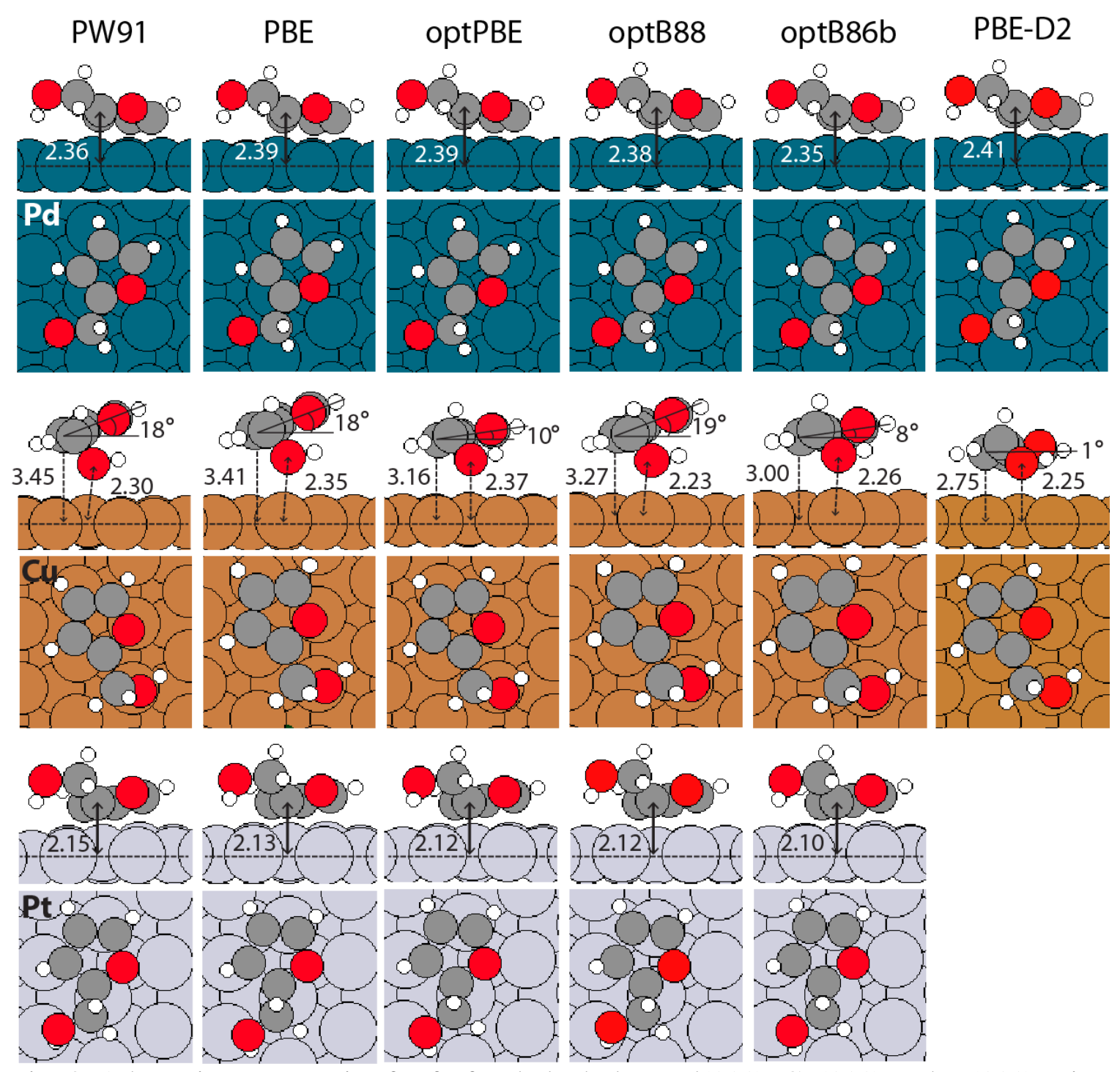

Fig. 3. Adsorption geometries for furfuryl alcohol on $\mathrm{Pd}(111), \mathrm{Cu}(111)$ and $\mathrm{Pt}(111)$ using PW91, PBE, optPBE, optB88, optB86b, and PBE-D2 functionals. Grey, white, and red spheres represent $\mathrm{C}, \mathrm{H}$, and $\mathrm{O}$ atoms, respectively. On $\mathrm{Pd}(111)$, perpendicular distances of furan ring to the surface are shown. $\mathrm{On} \mathrm{Cu}(111)$, the distances between the $\mathrm{O}$ atom in the $-\mathrm{CH}_{2} \mathrm{OH}$ group and the nearest $\mathrm{Cu}$ atom are given. On $\mathrm{Pt}(111)$, perpendicular distances of the furan ring to the surface are depicted.

On $\mathrm{Cu}(111)$, furfuryl alcohol adsorbs at the top site via the hydroxyl group in a cis configuration, which is consistent with its gas phase structure. Similar to the case of furfural adsorption, the perpendicular distances and the tilting angle of the ring vary with the choice of functional, where the furan ring is almost parallel to the $\mathrm{Cu}(111)$ surface as shown in Fig. 3. The binding energy increases for all the vdW-DF functionals compared to the GGA functionals, with the greatest increase observed for optB86b.

Finally, we see that the adsorption geometries for furfuryl alcohol on $\operatorname{Pt}(111)$ are similar to the corresponding geometries on $\operatorname{Pd}(111)$. The trends in binding energies are 
very similar to the corresponding trends on $\operatorname{Pd}(111)$, with furfuryl alcohol generally showing stronger binding on $\operatorname{Pt}(111)$ compared to $\operatorname{Pd}(111)$.

In summary, dispersion contributions have very small effects on the geometries of furfural and furfuryl alcohol adsorption on $\operatorname{Pd}(111)$ and $\operatorname{Pt}(111)$, with slightly larger effects observed on $\mathrm{Cu}(111)$. Corresponding changes in binding energies are much more significant, with increases of $1 \mathrm{eV}$ being typical of many of the dispersion functionals as compared to the GGA functionals.

A number of selected furfural hydrogenation intermediates, including monohydrogenation intermediates, and higher hydrogenation intermediates, have also been studied with explicit DFT calculations using both GGA (PW91, PBE) and vdW-DF (optB86b) functionals. Their most stable adsorption geometries, and the corresponding binding energies, are reported in the Supporting Information.

\subsection{Scaling relationship for binding energies}

The furfural hydrogenation reaction network contains a large number of hydrogenation intermediates, and explicit DFT investigations of all possible intermediates on multiple surfaces are computationally intensive. A bond-order based scaling correlation, with parameters determined by comparison to the results of selected DFT calculations, has been developed in a separate study for the estimation of the binding energies of glycerol dehydrogenation intermediates on transition metals, and this strategy has been successfully used to efficiently analyze a comparably large reaction network [14, 46-49]. To facilitate future studies of furfural reaction networks on transition metal surfaces, we briefly present a similar correlation based on the results of the calculated binding energies described in the previous section and in the Supporting Information.

Using gas phase $\mathrm{H}_{2}$, furfural, and the clean surface as reference states, $B E_{C_{5} H_{x} O_{2}}$ is defined in Eqn. (1), where $x$ represents the number of $\mathrm{H}$ atoms in the hydrogenation intermediate. $E_{C_{5} H_{x} O_{2}}, E_{*}, E_{C_{5} H_{4} O_{2}(g)}$, and $E_{H_{2}(g)}$, in turn, represent the total energy of adsorbed $\mathrm{C}_{5} \mathrm{H}_{\mathrm{x}} \mathrm{O}_{2}$, the total energy of clean surface, the total energy of gas phase furfural, and the total energy of gas phase $\mathrm{H}_{2}$, respectively.

$$
\begin{gathered}
B E_{C_{5} H_{x} O_{2}}=E_{C_{5} H_{x} O_{2}} \quad E_{*} \quad E_{C_{5} H_{4} O_{2}(g)} \frac{(x 4}{2} E_{H_{2}(g)} \\
v_{i}=\frac{n_{H \max } n_{\text {bond }}}{n_{H \max }}
\end{gathered}
$$

The fundamental assumption of this scaling relationship is that the binding energies for the unsaturated $\mathrm{C}$ and $\mathrm{O}$ atoms in the molecule are proportional to their respective valences $\left(v_{i}\right)$, which are defined by Eqn. (2), where $n_{H_{\text {max }}}$ is the maximum number of $\mathrm{H}$ 
atoms for $\mathrm{C}\left(n_{H_{\max }}=4\right)$ and $\mathrm{O}\left(n_{H_{\max }}=2\right)$, and $n_{\text {bond }}$ is the number of non-surface bonds for the $\mathrm{C}$ and $\mathrm{O}$ atoms in the given intermediate. The binding energy, $B E_{C_{5} H_{x} O_{2}}$, is given by Eqn. (3):

$$
B E_{C_{5} H_{x} O_{2}}=p_{i=2,5} v_{C i}+p_{i=3,4,6} p_{C 2} v_{C i}+p_{O} v_{O 7}+p_{C O} v_{C 6} v_{O 7}+p_{i, j=26} p_{C C} v_{C i} v_{C j}+p^{o}
$$

$p^{o}$ represents a constant parameter that includes the binding energy of furfural on $\operatorname{Pd}(111)$ (c.f. Table 2). $p_{C 1}$ and $p_{C 2}$ are first-order parameters for the C2/C5 atoms (see Fig. 1 for index assignment) and the C3/C4/C6 atoms, respectively. $p_{O}$ is the first-order parameter for the $\mathrm{O} 7$ atom, $p_{C O}$ is a second-order correlation parameter for $\mathrm{C} 6-\mathrm{O} 7$ pair in the aldehyde group, and $p_{C C}$ is a second-order correlation parameter for all $\mathrm{C}-\mathrm{C}$ pairs. The groupings of $\mathrm{C} 2 / \mathrm{C} 5$ and $\mathrm{C} 3 / \mathrm{C} 4 / \mathrm{C} 6$ for the first-order parameters are largely motivated by physical considerations; $\mathrm{C} 2 / \mathrm{C} 5$ and $\mathrm{C} 3 / \mathrm{C} 4$ are symmetrically distinct species, with the $\mathrm{C} 2 / \mathrm{C} 5$ atoms both being directly bonded to the etheric oxygen and the $\mathrm{C} 3 / \mathrm{C} 4$ atoms bonded only to other carbon atoms. Although similar physical considerations might argue that $\mathrm{C} 6$ should be fit with a separate first-order parameter, we found that this approach did not improve the quality of the fit, and to limit the total number of parameters, we used the same parameter for $\mathrm{C} 6$ as for $\mathrm{C} 3 / \mathrm{C} 4$ (lumping $\mathrm{C} 6$ with $\mathrm{C} 2 / \mathrm{C} 5$ gave very similar results). The six parameters are determined by fitting to the binding energies of 18 total species (furfural, furfuryl alcohol, and sixteen intermediates) based on DFT calculations. The binding energies of the remaining 16 intermediates are listed in Table $\mathrm{S} 3$. The parameters values are: $p_{C 1}=2.05, p_{C 2}=2.56$, $p_{O}=1.55, p_{C O}=-3.12, p_{C C}=-3.30$, and $p^{o}=-4.00$. We note that this correlation is quite similar to the correlation introduced previously for linear polyols although some differences are needed to account for the ring-like nature of the furfural derivatives. 


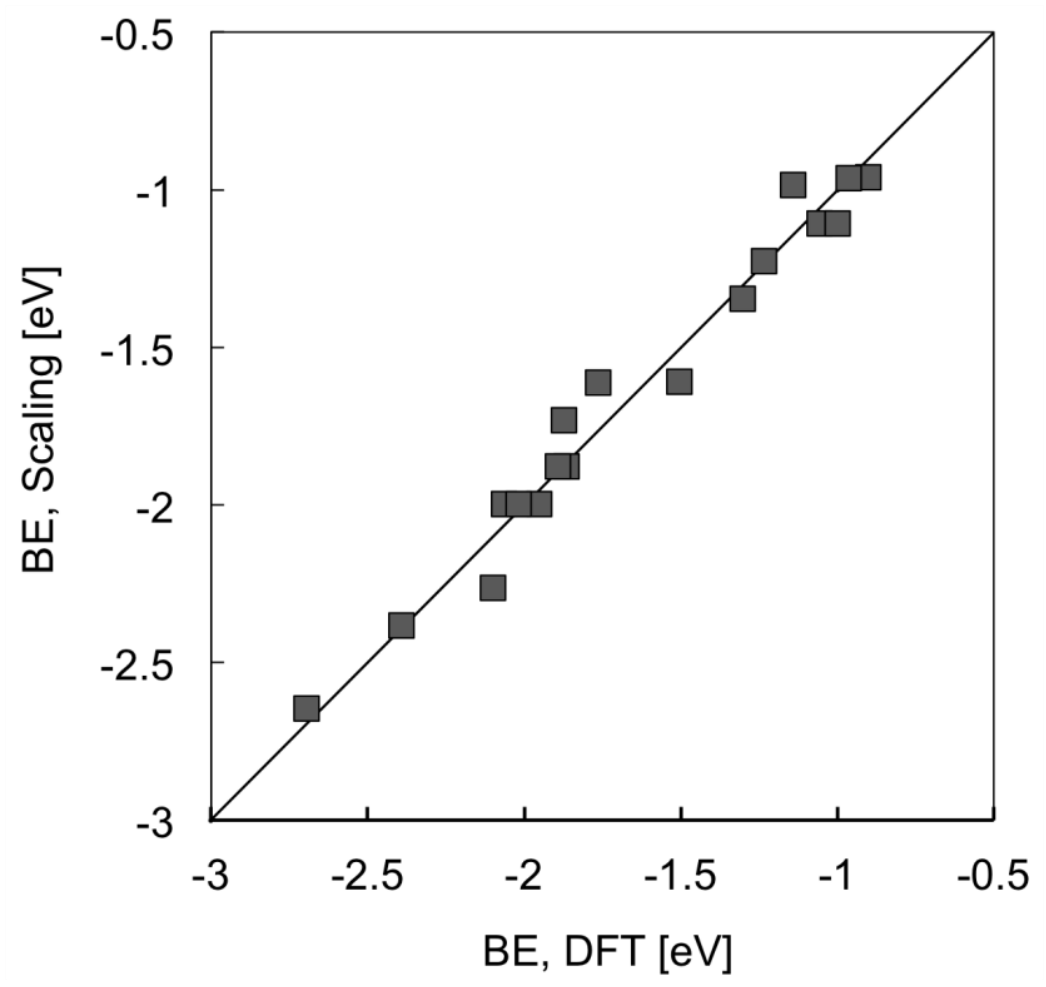

Fig. 4. Comparison of the binding energies from DFT calculations (PW91 functional) and the binding energies predicted with Eqn. (3) for furfural and selected furfural hydrogenation intermediates on $\operatorname{Pd}(111)$. The parity line is included as a guide to the eye. The standard error for the fit is $0.09 \mathrm{eV}$.

Fig. 4 compares the binding energies from DFT calculations using the PW91 functional and the binding energies predicted with Eqn. (3) for furfural and its hydrogenation intermediates on $\mathrm{Pd}(111)$. A reasonable level of agreement has been achieved between the bond-order scaling relationship, i.e., Eqn. (3), and the DFT calculations, with a standard error of $0.09 \mathrm{eV}$ over the entire data range.

In addition to correlations for multiple intermediates on a given metal surface, it is possible to develop additional linear scaling relationships to relate the binding energies of hydrocarbon [50] and oxygenated hydrocarbon [47] species across different transition metals with reasonable accuracy:

$$
B E_{M}=B E_{P d}+v_{i} v_{C i}\left(B E_{C M} \quad B E_{C P d}\right)+v_{i} v_{O i}\left(B E_{O M} \quad B E_{O P d}\right)
$$

In Eqn. (4), the binding energy of a given intermediate, $B E_{M}$, on metal $M(\mathrm{Pd}, \mathrm{Cu}, \mathrm{Pt})$ is scaled against the corresponding values, $B E_{P d}$, using the binding energy values of atomic $\mathrm{C}, \mathrm{O}$ on the respective surfaces $\left(B E_{C-P d}, B E_{C-M}, B E_{O-P d}\right.$, and $\left.B E_{O-M}\right)$. These values are listed in Table 3. We note that the preferred binding sites are hcp, fcc, fcc for $\mathrm{C}$ and fcc, fcc, fcc for $\mathrm{O}$ on $\mathrm{Pd}(111), \mathrm{Cu}(111)$ and $\mathrm{Pt}(111)$, respectively. 
Table 3 Binding energies (in eV) of atomic $\mathrm{C}$ and $\mathrm{O}$ on $\mathrm{Pd}(111), \mathrm{Cu}(111)$, and $\mathrm{Pt}(111)$ using PW91, PBE, optPBE, optB88, and optB86b functionals.

\begin{tabular}{cccc|ccc}
\hline GGA & \multicolumn{3}{c|}{$\mathrm{C}$} & \multicolumn{3}{c}{$\mathrm{O}$} \\
\cline { 2 - 7 } functionals & $\mathrm{Pd}$ & $\mathrm{Cu}$ & $\mathrm{Pt}$ & $\mathrm{Pd}$ & $\mathrm{Cu}$ & $\mathrm{Pt}$ \\
\hline PW91 & -6.93 & -4.90 & -7.42 & -4.49 & -4.86 & -4.59 \\
PBE & -7.03 & -4.95 & -7.47 & -4.39 & -4.65 & -4.40 \\
\hline vdW-DF & & & & & & \\
functionals & & & & & & \\
optPBE & -6.83 & -4.82 & -7.18 & -4.47 & -4.80 & -4.42 \\
optB88 & -6.89 & -4.92 & -7.25 & -4.84 & -5.19 & -4.80 \\
optB86b & -7.10 & -5.12 & -7.43 & -4.71 & -5.00 & -4.66 \\
\hline
\end{tabular}




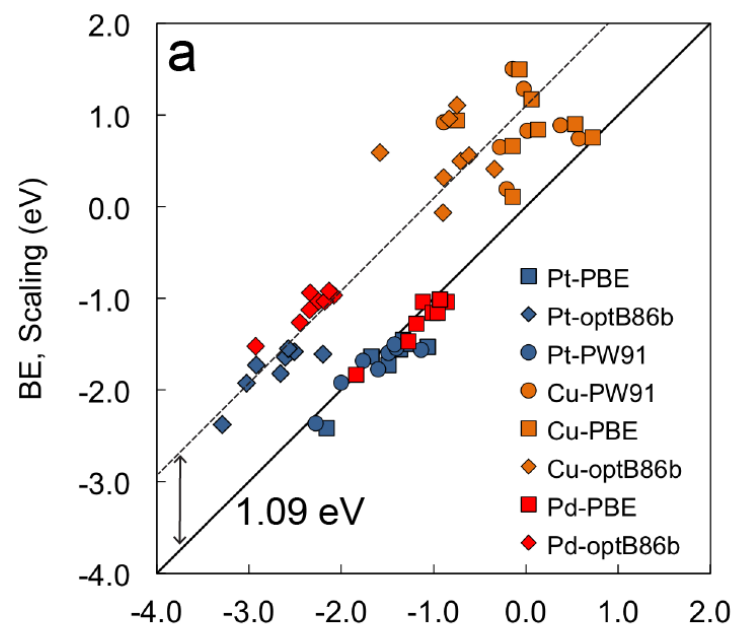

BE, Pd-PW91 (eV)

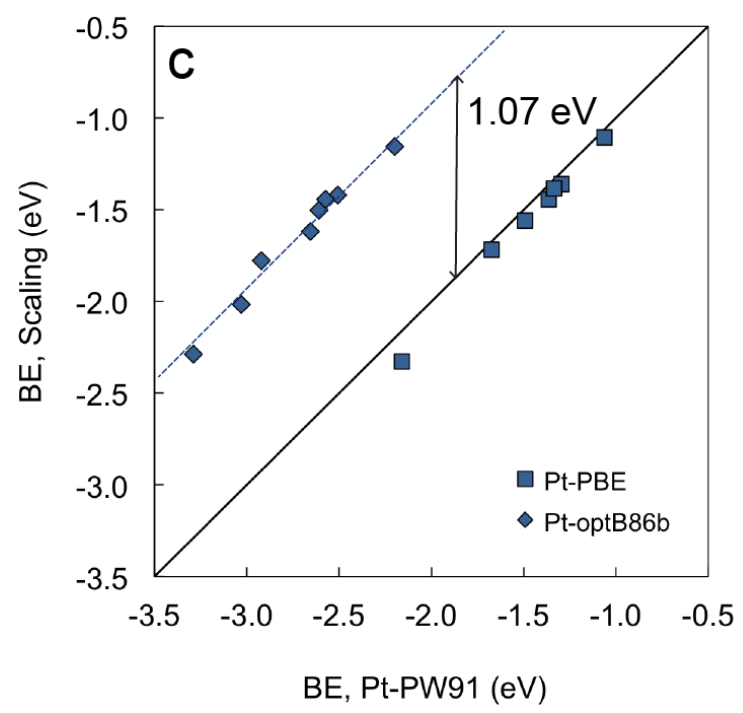

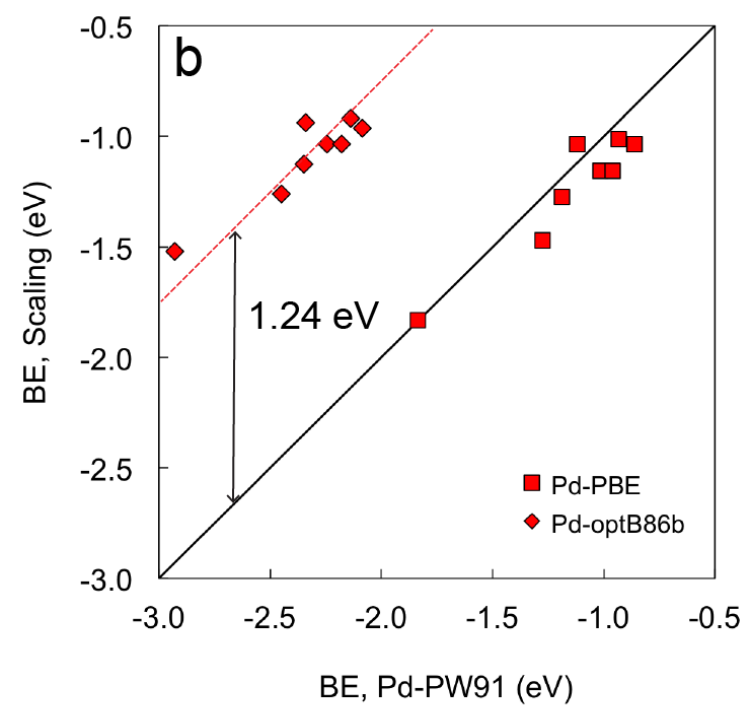

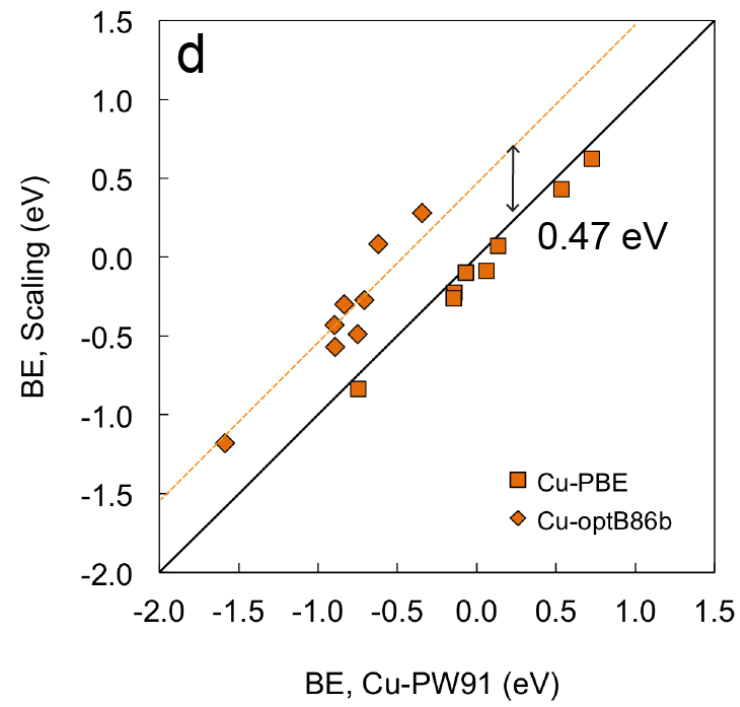

Fig. 5. (a) Binding energies of furfural, mono-hydrogenated intermediates of furfural, and furfuryl alcohol on $\mathrm{Pd}(111), \mathrm{Cu}(111)$, and $\mathrm{Pt}(111)$ surfaces for the PW91, PBE, and optB86b functionals, predicted using Eqn. (4) and the PW91 binding energies on $\mathrm{Pd}(111)$, (b) binding energies on the $\mathrm{Pd}(111)$ surface using PBE and optB86b functionals predicted using Eqn. (4) and the Pd(111)-PW91 binding energies (dashed line shows the average deviation, $1.24 \mathrm{eV}$, for optB86b), (c) binding energies on $\operatorname{Pt}(111)$ for the $\mathrm{PBE}$ and optB86b functionals predicted with Eqn. (4) and the Pt(111)-PW91 binding energies (dashed line shows the average deviation, $1.07 \mathrm{eV}$, for optB86b), (d) binding energies on the $\mathrm{Cu}(111)$ surface for the PBE and optB86b functionals predicted using Eqn. (4) and the $\mathrm{Cu}(111)-\mathrm{PW} 91$ binding energies (dashed line shows the average deviation, $0.47 \mathrm{eV}$, for optB86b). Parity lines are included as guides to the eye. The shifts of the VdW functionals from the parity lines are averaged over all calculated data points. 
Using the $\mathrm{C}$ and $\mathrm{O}$ binding energies tabulated in Table 3 and the binding energies of the furfural hydrogenation intermediates calculated for the Pd(111) surface with PW91 (reported in the Supporting Information), the binding energies of the same intermediates on the $\mathrm{Pt}(111)$ and $\mathrm{Cu}(111)$ surfaces, with the PW91, PBE and optB86b functionals, can also be estimated using Eqn. (4).

Fig. 5a shows the predicted binding energies of furfural, its mono-hydrogenated intermediates, and furfuryl alcohol on $\operatorname{Pd}(111), \mathrm{Cu}(111)$, and $\operatorname{Pt}(111)$ surfaces for the PW91, PBE and optB86b functionals, based on Eqn. (4) and the corresponding PW91 binding energies on Pd(111). The standard errors for Pt-PBE, Pt-optB86b, Pt-PW91, CuPW91, Cu-PBE, Cu-optB86b, Pd-PBE, and Pd-optB86b are $0.16 \mathrm{eV}, 0.16 \mathrm{eV}, 0.13 \mathrm{eV}$, $0.43 \mathrm{eV}, 0.39 \mathrm{eV}, 0.43 \mathrm{eV}, 0.10 \mathrm{eV}$ and $0.08 \mathrm{eV}$, respectively. It can be seen that good agreement between the predicted binding energies and the actual DFT calculations has been achieved for the Pd-PBE, Pt-PW91 and Pt-PBE calculations. This result strongly suggests that, in addition to its well-known use in extrapolating binding energies for a given functional between different metals, Eqn. (4) is also useful for extrapolating binding energies between different GGA functionals. However, we note that there is a constant shift for the predicted binding energies for the optB86b functional on $\operatorname{Pd}(111)$ and $\mathrm{Pt}(111)$ surfaces. The dashed line shows the combined average deviations between DFT calculations and scaling predictions (same for Fig. 5c and Fig. 5d), which is 1.09 $\mathrm{eV}$. This deviation indicates that Eqn. (4) cannot be directly used to extrapolate from standard GGA functionals to dispersion-corrected functionals; there remains an approximately constant, nonlocal contribution to the dispersion-corrected binding energies that cannot be fully described as the sum of the individual atomic contributions alone. Finally, we note that Fig. 5a clearly indicates that the scaling relationship based on Eqn. (4) completely fails for $\mathrm{Cu}(111)$. Due to the weak interactions between the various furfural derivatives and the $\mathrm{Cu}(111)$ surface, the underlying valency rule is not rigorously satisfied.

Fig. 5b-d show separate scaling plots for the different metals, where the predicted binding energies are based on the atomic $\mathrm{C}$ and $\mathrm{O}$ binding energies (PW91) calculated separately on the respective $\operatorname{Pd}(111), \operatorname{Pt}(111)$ and $\mathrm{Cu}(111)$ surfaces. These plots further confirm that, while excellent extrapolations can be made between the GGA functionals, constant offsets due to nonlocal contributions exist for the dispersion-corrected functionals. The respective shifts are $1.24 \mathrm{eV}$ for $\mathrm{Pd}, 1.07 \mathrm{eV}$ for $\mathrm{Pt}$, and $0.47 \mathrm{eV}$ for $\mathrm{Cu}$, respectively, with standard errors being $0.08 \mathrm{eV}$ and 0.10 for Pd-PBE and Pd-optB86b; $0.03 \mathrm{eV}$ and $0.06 \mathrm{eV}$ for Pt-PBE and Pt-optB86b; and $0.04 \mathrm{eV}$ and $0.14 \mathrm{eV}$ for $\mathrm{Cu}-\mathrm{PBE}$ and $\mathrm{Cu}-\mathrm{optB} 86 \mathrm{~b}$, in panels b-d respectively. 


\subsection{Brønsted-Evans-Polanyi relationship for furfural hydrogenation}

Brønsted-Evans-Polanyi (BEP) relationships provide an efficient means of approximating elementary reaction energy barriers without explicit transition state searches in heterogeneous catalysis [16]. They have been widely used to quickly estimate reaction kinetics in detailed mechanistic studies [51] and in trends-based analyses [47]. In this spirit, a BEP relationship can be developed for hydrogenation reactions associated with the intermediates discussed in the previous sections.

Table 4 gives the elementary reactions selected for the development of the BEP relationship, which is in turn shown in Fig. 6. The energy barriers of all the 13 elementary steps have been explicitly calculated using the CINEB and dimer methods with the PW91 and optB86b functionals on $\mathrm{Pd}(111)$. For $\mathrm{Cu}(111)$ and $\mathrm{Pt}(111)$, however, only the reactions relevant to furfural alcohol formation are reported. Transition state geometries can be found in the Supporting Information. Relevant gas phase species used as references states of the elementary steps were calculated within the same computational framework and respective functionals (i.e., PW91 or optB86b).

Table 4. Energy barriers (in $\mathrm{eV}$ ) for selected hydrogenation reactions. FA* represents adsorbed furfuryl alcohol. $\operatorname{mh} n^{*}(n=2,3,4,5,6,7)$ represents mono-hydrogenated intermediates, and $n$ represents the site index defined in Fig. 1.

\begin{tabular}{|c|c|c|c|c|c|c|c|}
\hline & \multirow{2}{*}{ Reactions } & \multicolumn{2}{|c|}{$\operatorname{Pd}(111)$} & \multicolumn{2}{|c|}{$\mathrm{Cu}(111)$} & \multicolumn{2}{|c|}{$\operatorname{Pt}(111)$} \\
\hline & & PW91 & optB86b & PW91 & optB86b & PW91 & optB86b \\
\hline 1 & furfural $*+\mathrm{H}^{*} \rightarrow \mathrm{mh} 2 *$ & 1.05 & 1.12 & --- & --- & --- & --- \\
\hline 2 & furfural $*+\mathrm{H}^{*} \rightarrow \mathrm{mh} 3 *$ & 1.02 & 0.94 & --- & --- & --- & --- \\
\hline 3 & furfural $*+\mathrm{H}^{*} \rightarrow \mathrm{mh} 4^{*}$ & 1.04 & 0.95 & --- & --- & --- & --- \\
\hline 4 & furfural $*+\mathrm{H}^{*} \rightarrow \mathrm{mh} 5^{*}$ & 0.96 & 0.92 & --- & --- & --- & --- \\
\hline 5 & furfural $*+\mathrm{H}^{*} \rightarrow \mathrm{mh}^{*}$ & 0.96 & 0.83 & 0.56 & 0.46 & 0.52 & 0.38 \\
\hline 6 & furfural $*+\mathrm{H}^{*} \rightarrow \mathrm{mh} 7 *$ & 0.71 & 0.64 & 0.74 & 0.71 & 0.48 & 0.40 \\
\hline 7 & furfural $* \rightarrow \mathrm{C}_{5} \mathrm{H}_{3} \mathrm{O}_{2} *+\mathrm{H}^{*}$ & 0.99 & 0.95 & --- & --- & --- & --- \\
\hline 8 & $\mathrm{mh}^{*}+\mathrm{H}^{*} \rightarrow \mathrm{FA}^{*}$ & 0.59 & 0.51 & 1.15 & 1.11 & 0.61 & 0.65 \\
\hline 9 & $\mathrm{mh}^{*}+\mathrm{H}^{*} \rightarrow \mathrm{FA}^{*}$ & 0.91 & 0.88 & 0.24 & 0.39 & 1.12 & 1.12 \\
\hline 10 & $\mathrm{FA}^{*}+\mathrm{H}^{*} \rightarrow$ th $267^{*}$ & 0.93 & 0.91 & --- & --- & --- & --- \\
\hline 11 & $\mathrm{FA}^{*}+\mathrm{H}^{*} \rightarrow$ th $367^{*}$ & 1.16 & 1.01 & --- & --- & --- & --- \\
\hline 12 & $\mathrm{FA}^{*}+\mathrm{H}^{*} \rightarrow$ th $467^{*}$ & 1.17 & 1.09 & --- & --- & --- & --- \\
\hline 13 & FA* $+\mathrm{H}^{*} \rightarrow$ th567* & 0.97 & 0.83 & --- & --- & --- & --- \\
\hline
\end{tabular}

Most of the energy barriers calculated from the optB86b functional in Table 6 are very similar to the corresponding barriers calculated with the PW91 functional. Calculations using both functionals show that the hydrogenation of $\mathrm{O} 7$ (Rxns. 6 and 8) is kinetically more facile than C6 (Rxns. 5 and 9) on $\operatorname{Pd}(111)$ and $\operatorname{Pt}(111)$, which is in turn opposite to the trend on $\mathrm{Cu}(111)$. 
Fig. 6 shows the corresponding BEP relationship established between the transition states and final states. $E_{F S}$ represents the final state energy for an elementary step written in the exothermic direction, using the gas phase reactants as the reference. $E_{T S}$ represents the corresponding transition state energy. The square symbols represent PW91 calculations, and the diamond symbols represent optB86b calculations. The straight line is the least squares fit using only the Pd(111)-PW91 data, where the slope and the intercept are 1.01 (dimensionless) and $1.01(\mathrm{eV})$, respectively. The standard error obtained from the least squares fit is $0.13 \mathrm{eV}$. Clearly, this BEP line describes the other metals and the dispersion-corrected functionals quite well, strongly implying that the BEP relationship developed for standard GGA functionals (PW91 or PBE) is equally capable of describing reaction energetics for dispersion-corrected functionals.

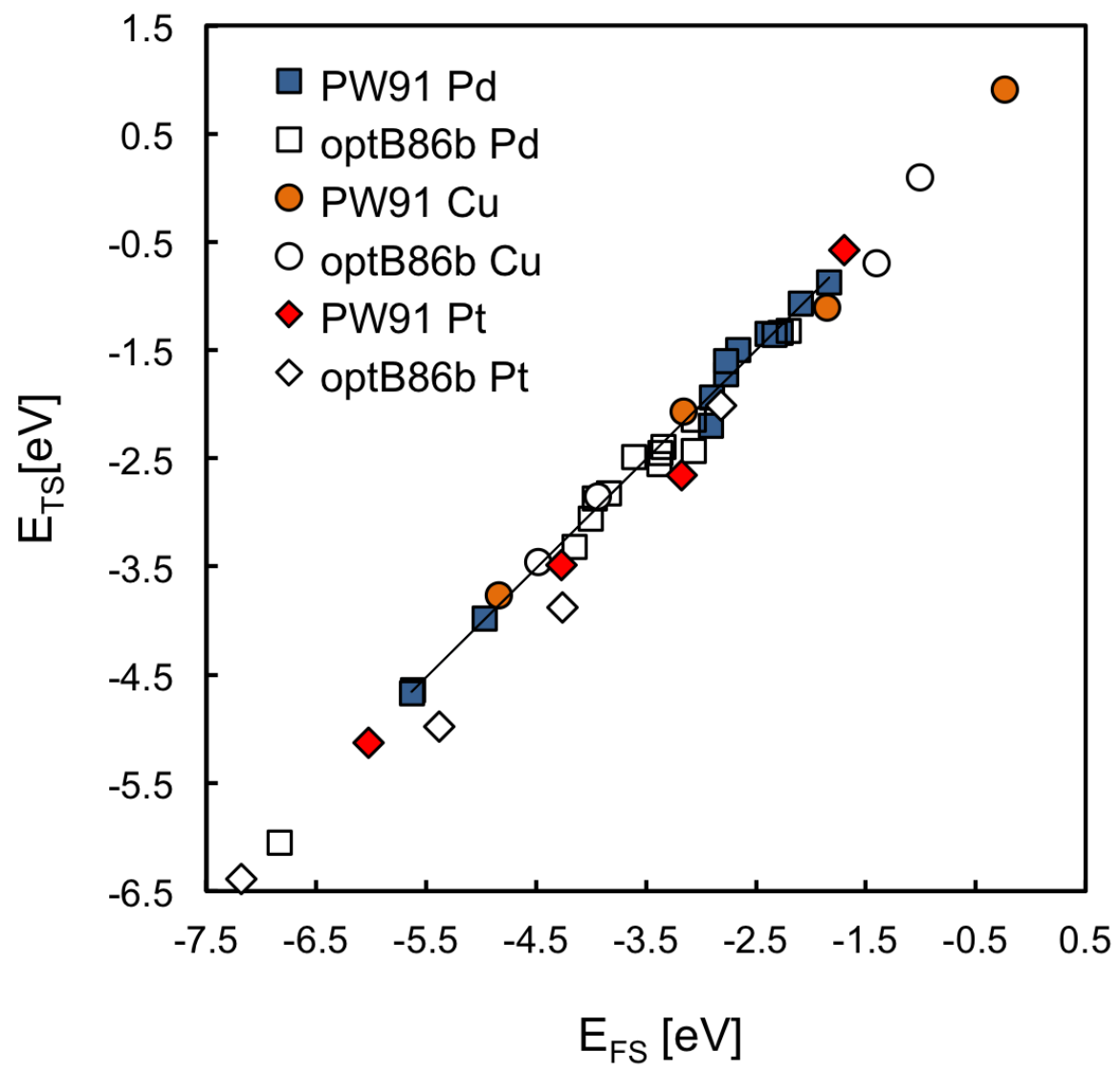

Fig. 6. The BEP relationship developed for $\mathrm{Pd}(111), \mathrm{Pt}(111)$ and $\mathrm{Cu}(111)$ using PW91 and optB86b functionals. $E_{F S}$ represents the final state energy for an elementary step written in the exothermic direction, using the gas phase reactants as the reference. $E_{T S}$ represents the corresponding transition state energy. The square symbols represent PW91 calculations and the diamond symbols represent optB86b calculations. The straight line is obtained from the least-square fit to Pd(111)-PW91 only. The slope and intercept of the least-square fit are 1.01 (dimensionless) and $1.01(\mathrm{eV})$, respectively. The standard error of the fit is 0.13 $\mathrm{eV}$. 


\subsection{Hydrogenation of furfural on $\operatorname{Pd}(111), \mathrm{Cu}(111)$ and $\operatorname{Pt}(111)$}

The thermodynamic and kinetic information described in the previous sections allows us to obtain some very simple insights into the influence of dispersion effects on hydrogenation reactions. Fig. 7 presents the potential energy surface for furfural hydrogenation to furfuryl alcohol on $\operatorname{Pd}(111), \mathrm{Cu}(111)$, and $\operatorname{Pt}(111)$ using gas phase furfural, the clean metal surface, and adsorbed $\mathrm{H}$ as references.

On $\operatorname{Pd}(111)$ and $\operatorname{Pt}(111)$, the adsorption structures are not substantially affected by the dispersion interactions, while on $\mathrm{Cu}(111)$, some geometric changes are observed. However, due to the contributions from van der Waals interactions, the binding energies become stronger on all metal surfaces. In effect, as is shown in Fig. 7, dispersion effects shift the potential energy surfaces for hydrogenation by an approximately constant amount, while relative energetics and reaction barriers between competing hydrogenation pathways are much less significantly affected. Although we cannot exclude the possibility that more substantial changes in barriers might be found for other reaction networks, it appears that, at least for these specific pathways, the impact of dispersion effects may thus be limited primarily to changes in surface coverages from corresponding binding energy changes in adsorption/desorption equilibria, while surface rate constants are largely unchanged. These considerations, in turn, imply that trends in reactivity across transition metals are not substantially altered when dispersion interactions are included, but quantitative predictions of rates may well be impacted.

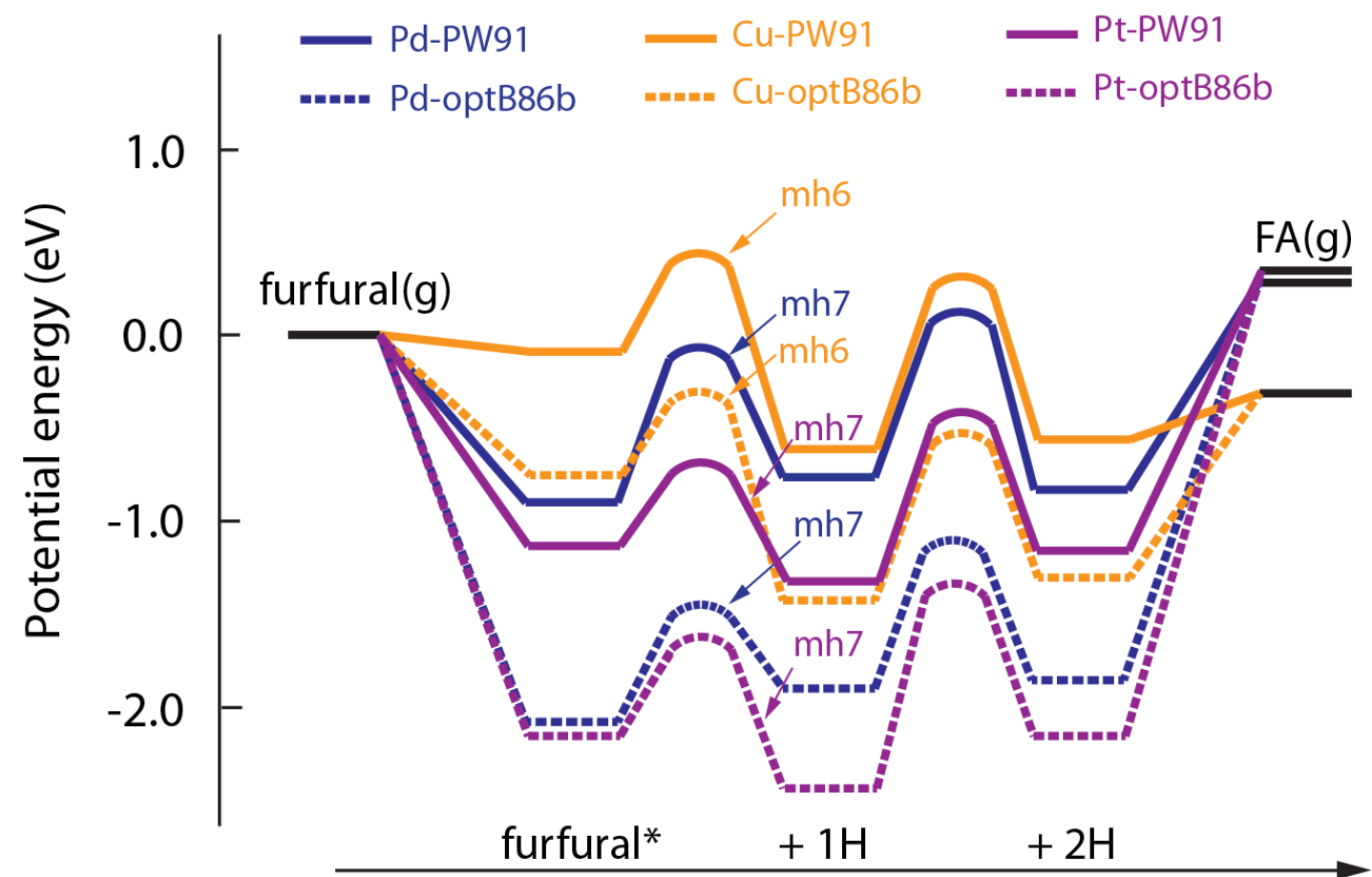

Fig. 7. Potential energy surface for lowest energy furfural hydrogenation pathways to furfuryl alcohol on $\mathrm{Pd}(111), \mathrm{Cu}(111)$ and $\mathrm{Pt}(111)$ surfaces using PW91 and optB86b functionals. The favored reaction pathway and hydrogenation intermediate 
for each metal are labeled and represented by bold lines.

\section{Conclusions}

The hydrogenation of furfural to furfuryl alcohol was used as a case study to elucidate some of the effects of dispersion in Density Functional Theory calculations involving unsaturated organic hydrocarbon species on transition metal surfaces. A number of common functionals from the vdW-DF family, including, optPBE, optB88, optB86b, as well as Grimme's DFT-D2/D3 method, were employed within a planewave, periodic DFT framework. The adsorption of furfural, partially hydrogenated furfural intermediates, and furfuryl alcohol on $\mathrm{Pd}(111), \mathrm{Cu}(111), \operatorname{Pt}(111)$ was analyzed with these functionals. The geometric features of furfural and furfuryl alcohol adsorption did not change significantly on $\operatorname{Pd}(111)$ and $\operatorname{Pt}(111)$, while modest geometric changes were observed on $\mathrm{Cu}(111)$. In contrast, increases in the magnitudes of binding energies (up to $\sim 1 \mathrm{eV}$ ) were seen on all surfaces. It was further determined that standard scaling relationships, similar to those developed by Nørskov and coworkers [50], can accurately describe the GGA results on all of the metal surfaces. However, when comparing GGA and dispersion-corrected binding energies on these surfaces, it was found that there exists a constant shift in the scaling relationships that arises from the non-local effects of the dispersion corrections. Although the effect of dispersion corrections on adsorption thermochemistry can be significant, it was determined that dispersion effects do not significantly affect the hydrogenation barriers, and Brønsted-Evans-Polanyi (BEP) relationships developed for standard GGA functionals are fully capable of describing the corresponding kinetics of dispersion-corrected functionals. The combined thermodynamic and kinetic data therefore suggest that dispersion corrections lead to approximately solid downshifts in potential energy surfaces for furfural hydrogenation.

\section{Acknowledgements}

This work is also supported as part of the Institute for Atom-efficient Chemical Transformations (IACT), an Energy Frontier Research Center funded by the U.S. Department of Energy, Office of Science, Office of Basic Energy Sciences. Use of the Center for Nanoscale Materials (CNM) is supported by the Office of Science of the US Department of Energy under contract No. DE-AC02-06CH11357. B.L. acknowledges the financial support from Kansas State University. We also acknowledge grants of computer time from EMSL, a national scientific user facility located at Pacific Northwest National Laboratory, Argonne Laboratory Computing Resource Center (LCRC), and the National Energy Research Scientific Computing Center (NERSC) for providing us with additional computing resources. 


\section{References}

[1] J.N. Chheda, G.W. Huber, J.A. Dumesic, Angewandte Chemie-International Edition 46 (2007) 7164-7183.

[2] N. Merat, C. Godawa, A. Gaset, Journal of Chemical Technology and Biotechnology 48 (1990) 145-159.

[3] P. Claus, Top Catal 5 (1998) 51-62.

[4] H.S. Luo, H.I. Li, L. Zhuang, Chemistry Letters (2001) 404-405.

[5] W. Zhang, Y.L. Zhu, S. Niu, Y.W. Li, Journal of Molecular Catalysis a-Chemical 335 (2011) 71-81.

[6] S. Sitthisa, D.E. Resasco, Catalysis Letters 141 (2011) 784-791.

[7] S. Sitthisa, P. Trung, T. Prasomsri, T. Sooknoi, R.G. Mallinson, D.E. Resasco, J Catal 280 (2011) 17-27.

[8] D.C. Elliott, T.R. Hart, Energy \& Fuels 23 (2009) 631-637.

[9] S. Sitthisa, W. An, D.E. Resasco, J Catal 284 (2011) 90-101.

[10] C.M. Horiuchi, J.W. Medlin, Langmuir 26 (2010) 13320-13332.

[11] S.H. Pang, J.W. Medlin, Acs Catalysis 1 (2011) 1272-1283.

[12] F. Studt, F. Abild-Pedersen, T. Bligaard, R.Z. Sorensen, C.H. Christensen, J.K. Nørskov, Science 320 (2008) 1320-1322.

[13] J. Greeley, M. Mavrikakis, Journal of the American Chemical Society 126 (2004) 39103919.

[14] B. Liu, J. Greeley, J. Phys. Chem. C 115 (2011) 19702-19709.

[15] M. Mavrikakis, M.A. Barteau, Journal of Molecular Catalysis a-Chemical 131 (1998) 135-147.

[16] J.K. Nørskov, T. Bligaard, A. Logadottir, S. Bahn, L.B. Hansen, M. Bollinger, H. Bengaard, B. Hammer, Z. Sljivancanin, M. Mavrikakis, Y. Xu, S. Dahl, C.J.H. Jacobsen, J Catal 209 (2002) 275-278.

[17] S. Wang, B. Temel, J. Shen, G. Jones, L.C. Grabow, F. Studt, T. Bligaard, F. AbildPedersen, C.H. Christensen, J.K. Norskov, Catalysis Letters 141 (2011) 370-373.

[18] P. Ferrin, D. Simonetti, S. Kandoi, E. Kunkes, J.A. Dumesic, J.K. Nørskov, M. Mavrikakis, Journal of the American Chemical Society 131 (2009) 5809-5815.

[19] H. Margenau, Reviews of Modern Physics 11 (1939) 1-35.

[20] A. Bilic, J.R. Reimers, N.S. Hush, J Phys Chem B 106 (2002) 6740-6747.

[21] A. Bilic, J.R. Reimers, N.S. Hush, R.C. Hoft, M.J. Ford, Journal of Chemical Theory and Computation 2 (2006) 1093-1105.

[22] M. Dion, H. Rydberg, E. Schroder, D.C. Langreth, B.I. Lundqvist, Physical Review Letters 92 (2004).

[23] J. Klimes, D.R. Bowler, A. Michaelides, J Phys-Condens Mat 22 (2010).

[24] V.R. Cooper, Physical Review B 81 (2010).

[25] S. Grimme, Journal of Computational Chemistry 27 (2006) 1787-1799.

[26] K. Tonigold, A. Gross, Journal of Chemical Physics 132 (2010).

[27] V. Vorotnikov, G. Mpourmpakis, D.G. Vlachos, Acs Catalysis 2 (2012) 2496-2504.

[28] G. Kresse, J. Hafner, Physical Review B 47 (1993) 558-561.

[29] G. Kresse, J. Furthmuller, Physical Review B 54 (1996) 11169-11186.

[30] P.E. Blochl, Physical Review B 50 (1994) 17953-17979.

[31] G. Kresse, D. Joubert, Physical Review B 59 (1999) 1758-1775.

[32] M. Methfessel, A.T. Paxton, Physical Review B 40 (1989) 3616-3621.

[33] J. Klimes, D.R. Bowler, A. Michaelides, Physical Review B 83 (2011). 
[34] J.P. Perdew, J.A. Chevary, S.H. Vosko, K.A. Jackson, M.R. Pederson, D.J. Singh, C. Fiolhais, Physical Review B 46 (1992) 6671-6687.

[35] J.P. Perdew, K. Burke, M. Ernzerhof, Physical Review Letters 77 (1996) 3865-3868.

[36] H.J. Monkhorst, J.D. Pack, Physical Review B 13 (1976) 5188-5192.

[37] G. Henkelman, G. Johannesson, H. Jonsson, in, Kluwer Academic, New York, 2000, pp. 269.

[38] G. Henkelman, H. Jonsson, Journal of Chemical Physics 113 (2000) 9978-9985.

[39] G. Henkelman, H. Jonsson, Journal of Chemical Physics 111 (1999) 7010-7022.

[40] R.A. Olsen, G.J. Kroes, G. Henkelman, A. Arnaldsson, H. Jonsson, Journal of Chemical Physics 121 (2004) 9776-9792.

[41] C. Kittel, Introduction to Solid State Physics, 7th ed., John Wiley \& Sons, 1996.

[42] T.S. Little, J. Qiu, J.R. Durig, Spectrochimica Acta Part a-Molecular and Biomolecular Spectroscopy 45 (1989) 789-794.

[43] S. Sitthisa, T. Sooknoi, Y.G. Ma, P.B. Balbuena, D.E. Resasco, J Catal 277 (2011) 1-13.

[44] S.H. Pang, A.M. Roman, J.W. Medlin, J. Phys. Chem. C 116 (2012) 13654-13660.

[45] M.J. Knight, F. Allegretti, E.A. Kroger, M. Polcik, C.L.A. Lamont, D.P. Woodruff, Surface Science 602 (2008) 2524-2531.

[46] B. Liu, J. Greeley, Top Catal 55 (2012) 280-289.

[47] B. Liu, J. Greeley, Phys Chem Chem Phys 15 (2013) 6475-6485.

[48] F. Mehmood, R.B. Rankin, J. Greeley, L.A. Curtiss, Phys Chem Chem Phys 14 (2012) 8644-8652.

[49] R.B. Rankin, J. Greeley, Acs Catalysis 2 (2012) 2664-2672.

[50] F. Abild-Pedersen, J. Greeley, F. Studt, J. Rossmeisl, T.R. Munter, P.G. Moses, E. Skulason, T. Bligaard, J.K. Nørskov, Physical Review Letters 99 (2007) 016105-016101.

[51] D. Loffreda, F. Delbecq, F. Vigne, P. Sautet, Angew Chem Int Ed Engl 48 (2009) 89788980. 